\title{
Enhancement of Oral Bioavailability of Puerarin by Polybutylcyanoacrylate Nanoparticles
}

\author{
Lixia Zhao, ${ }^{1,2}$ Anchang Liu, ${ }^{1,2}$ Min Sun,, 3 Jinsong Gu, ${ }^{4}$ Haigang Wang, ${ }^{2}$ Shuang Wang, \\ Jing Zhang, ${ }^{1}$ Chenyu Guo, ${ }^{1}$ Rui Duan, ${ }^{1}$ and Guangxi Zhai ${ }^{1}$ \\ ${ }^{1}$ School of Pharmaceutical Sciences, Shandong University, Jinan 250012, China \\ ${ }^{2}$ Department of Pharmacy, Qilu Hospital of Shandong University, Jinan 250012, China \\ ${ }^{3}$ Deparment of Pharmacy, Central Hospital of Zibo, Zibo 255036, China \\ ${ }^{4}$ Department of Biotechnology, College of Life Science and Technology, University of Jinan, Jinan 250022, China
}

Correspondence should be addressed to Guangxi Zhai, professorgxzhai@yahoo.cn

Received 14 June 2011; Accepted 6 July 2011

Academic Editor: Daxiang Cui

Copyright ( $) 2011$ Lixia Zhao et al. This is an open access article distributed under the Creative Commons Attribution License, which permits unrestricted use, distribution, and reproduction in any medium, provided the original work is properly cited.

The interest using novel drug delivery systems to improve oral bioavailability of drug with poor solubility is increasing. In this study, a new oral delivery system, polybutylcyanoacrylate nanoparticles (PBCNs), was introduced to improve the oral bioavailability of puerarin (PUE). PUE-loaded PBCN was successfully prepared by anionic polymerization method. Characterization of PUE-loaded PBCN was evaluated with morphology, size, zeta potential, and in vitro release study. The PBCN loading PUE exhibited a spherical shape under transmission electron microscopy with an average size of $159.4 \mathrm{~nm}$, and the zeta potential was $-15.0 \mathrm{mV}$. The in vitro release of PUE-loaded PBCN showed an initial burst release followed by a sustained release. Physicochemical state of PUE in PBCN was investigated by differential scanning colorimetry, X-ray diffraction, and Fourier transform infrared spectroscopy. The results indicated that PUE in PBCN was in a noncrystalline state. The oral pharmacokinetic study in rats showed that the relative bioavailability of PUE-encapsulated PBCN to the crude PUE was more than 550\%. It can be concluded that PBCN as an oral drug carrier can significantly improve the oral bioavailability of PUE.

\section{Introduction}

The bioavailability is an important parameter showing the degree and rate of drug molecules entering blood circulation, indicating the effectiveness and safety of an extravascular administration formulation. It can be influenced by drug formulations, food, and physiological factors. However, up to $50 \%$ of orally administered drugs present formulation problems related to poor solubility $(0.011 \mathrm{M})$ [1]. In recent years, many specific pharmaceutical approaches such as micro- and nanotechnology have been developed to improve the bioavailability [2]. As one promising delivery system with improved bioavailability, polybutylcyanoacrylate nanoparticles (PBCNs) have attracted considerable attention [3]. It is a type of solid colloidal particles ranging in size from $10 \mathrm{~nm}$ to $1000 \mathrm{~nm}$ made of biodegradable polymers $[4,5]$ which could significantly enhance the oral absorption of some drugs such as thymopentin [6]. The PBCNs that served as the oral carriers can prevent the destruction of drugs (e.g., peptide drugs) by the acid and enzymes in gastrointestinal tract $[7,8]$, improve the drug absorption through Peyer's patches (PP), the immunization-related tissue in small intestine of human and animals which accounts for about $25 \%$ of the intestinal mucosa, and other intestinal lymphoid tissue into the blood circulation [9], and prolong the residence time in vivo because of the small particles and bioadhesive property [6].

Puerarin (PUE, Figure 1) is the naturally occurring isoflavone C-glycoside extracted from the roots of Pueraria lobata (Willd.), Ohwi, and P. thomsonni Benth, which is widely used in China for the treatment of cerebrovascular and cardiovascular disease. Although PUE has definite therapeutic effectiveness, its clinical application is limited by its poor solubility, short elimination half-life, and poor oral bioavailability. The commercial available preparation for PUE is its injection which has poor patient compliance 


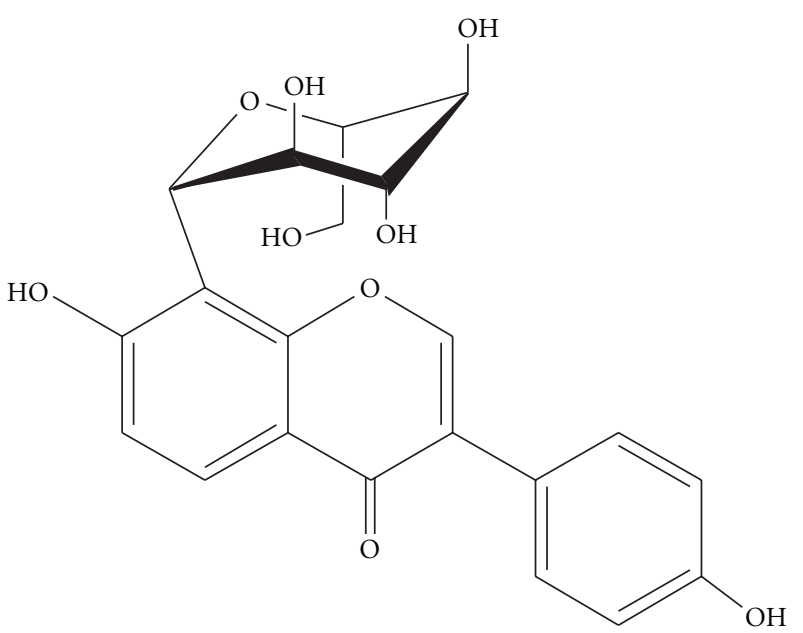

(a)

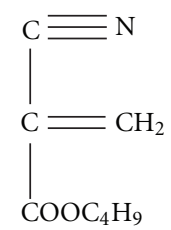

(b)

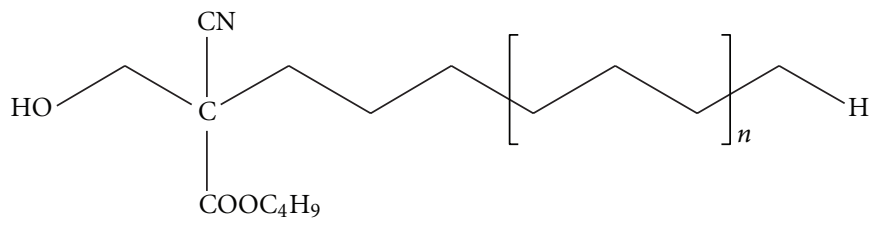

(c)

FIgure 1: Chemical structure of puerarin (a), BCA (b) and PBCA (c).

because of the high frequency of injecting administration [10]. The oral preparation containing Pueraria lobata extraction is Yufengningxin tablet which needs to be orally given five tables each time and three times each day. Therefore, the development of a suitable oral preparation is a research focus of PUE. In the past few years, several pharmaceutical techniques were adopted to improve the oral absorption of puerarin, such as microemulsion [10-12] and solid lipid nanoparticles [13]. However, up to date no study on PBCN for enhancing the oral bioavailability of PUE has been reported.

In this study, PUE-loaded PBCN (PUE-PBCN) was prepared by anionic polymerization method in order to improve the oral bioavailability of PUE and was characterized with morphology, size, zeta potential, and in vitro release study. Physicochemical state of PUE in PBCN was investigated by differential scanning colorimetry (DSC), X-ray diffraction (XRD), and Fourier transform infrared (FTIR) spectroscopy. Additionally, the pharmacokinetics of PUE-loaded PBCN was studied in rats.

\section{Materials and Methods}

2.1. Materials. PUE was purchased from Nanjing Zelang Medical Technology Co., Ltd. (Nanjing, China). Dextran 70 (D-(+) glucose) and poloxamer 188 (F68) were provided by the Shanghai Treechem Biotech Co., Ltd. (Shanghai, China). N-Butylcyanoacrylate (BCA) was a gift sample from Beijing Suncon Medical Adhesive Co., Ltd. (Beijing, China). All other reagents used in the study were of analytical regent grade.
2.2. Preparation of $P B C N$. Based on the previous reports with some modifications [14-16], PUE-PBCN was prepared using open anionic polymerization of n-butyl-2cyanoacrylate (BCA) monomer in acidic medium $(0.01 \mathrm{M}$ $\mathrm{HCl})$, containing $0.5 \%(\mathrm{w} / \mathrm{v})$ of dextran 70 and $0.5 \%(\mathrm{w} / \mathrm{v})$ of poloxamer 188 as stabilizer and emulsifier. The monomer BCA was injected drop by drop into the stirred medium including PUE (the weight ratio of drug to polymer was $1: 2$ ). Polymerization was conducted for 4 hours under magnetic agitation at $400 \mathrm{rpm}$ and room temperature. Subsequently, sodium hydroxide solution $(1 \mathrm{M})$ was used to adjust the $\mathrm{pH}$ of resulting suspension to $6.8 \pm 0.1$. The above mixture was stirred for an additional $1 \mathrm{~h}$ at the same conditions, and the reaction was gradually completed. The PUE-PBCN suspension was obtained after the above system was filtered through $0.8 \mu \mathrm{m}$ filter membrane.

\subsection{Characterization of $P B C N$}

2.3.1. Morphology. PUE-loaded PBCN was observed under a transmission electron microscope (TEM, JEM-1200EX, JEOL, Tokyo, Japan) using the negative-staining method. One drop of diluted PUE-PBCN was added to a copper grid to form a thin liquid film, and then the film was negatively stained by adding $2 \%(\mathrm{w} / \mathrm{v})$ phosphotungstic acid (PTA, $\mathrm{pH}$ 7.0). Extra droplet was instantly removed with filter paper, and then the grid was dried at room temperature as a TEM sample. The film was examined under TEM and photographed. 
2.3.2. Particle Size and Zeta Potential. The particle size of PUE-PBCN was analyzed using a particle sizer (Zetasizer 3000 HAS, Malvern Instruments Ltd., Malvern, Worcestershire, UK) with photon correlation spectroscopy (PCS) at a fixed angle of $90^{\circ}$ at a temperature of $25^{\circ} \mathrm{C}$. It was conducted with He-Ne laser of $3 \mathrm{~mW}$ at a wavelength of $633 \mathrm{~nm}$, and the particle size analysis data were evaluated using the volume distribution.

Zeta potential of PUE-PBCN was determined using TV microscopic electrophoresis system (DXD-II, Optics Co., Ltd., Jiangsu, China) at room temperature.

2.3.3. Encapsulation Efficiency (EE) and Drug Loading (DL). The measurement for encapsulation efficiency and drug loading of PUE-PBCN was carried out with centrifugation ultrafiltration method according to the previous reports $[17,18]$. The free PUE was separated from PUE-PBCN by centrifugal filter tubes (Amicon Ultra-4, Millipore, Ireland) with a molecular cut off of $10 \mathrm{kDa}$. Briefly, $2 \mathrm{~mL}$ of PUEloaded nanoparticle suspension was added into centrifugal filter tube and centrifuged at $4000 \mathrm{rpm}$ for $20 \mathrm{~min}$ at room temperature using a centrifuge (Biofuge primo R. Heraeus, Hanau, Germany). The initial total amount of PUE in the suspension system and the amount of free drug in the filtrate were measured using UV at the wavelength of $251 \mathrm{~nm}$. The encapsulation efficiency and drug loading in the PBCN were calculated as, respectively follows:

$$
\begin{aligned}
& \mathrm{EE} \%=\frac{W_{\text {total drug }}-W_{\text {free drug }}}{W_{\text {total drug }}} \times 100 \%, \\
& \mathrm{DL} \%=\frac{W_{\text {total drug }}-W_{\text {free drug }}}{W_{\text {polymer }}} \times 100 \%,
\end{aligned}
$$

where " $W_{\text {free drug }}$ " is the amount of PUE unloaded in PBCA nanoparticles, " $W_{\text {total drug }}$ " is the initial total amount of PUE in the suspension system, and " $W_{\text {polymer }}$ " is the weight of butyl-cyanoacrylate monomer.

2.3.4. DSC and XRD Analysis. The physical state of the drug entrapped in the $\mathrm{PBCN}$ was characterized using a differential scanning calorimeter (CDR-4P, Shanghai Tianping Instrument Ltd., Shanghai, China) and an X-ray diffractometer (D/max r-B, Rigaku Co., Tokyo, Japan). Prior to analysis, PUE-PBCN and blank PBCN used for DSC and XRD were obtained by freeze-drying without any freeze-dried protectants.

For DSC measurement, about $10 \mathrm{mg}$ of samples (PUE, PUE-PBCN, or blank PBCN) were sealed in the aluminum pan and heated at a scanning rate of $10^{\circ} \mathrm{C} / \mathrm{min}$ from 30 to $400^{\circ} \mathrm{C}$ under dry nitrogen atmosphere at a flow rate of $0.2 \mathrm{~mL} / \mathrm{min}$.

X-ray diffraction patterns were determined for PUEPBCN, blank PBCN, and cure PUE with a $\mathrm{Cu}$ line as the source of radiation. A radiation at $40 \mathrm{kV}$ voltage and $40 \mathrm{~mA}$ current was used, and diffractograms were performed with a scanning rate of $2^{\circ} / \mathrm{min}$ over a $2 \theta$ range of from $6^{\circ}$ to $40^{\circ}$.

2.3.5. Fourier Transform Infrared (FTIR) Spectroscopy. FTIR analysis was performed to provide further information on the drug-polymer relationship using an FTIR spectrometer (Thermo Electron Scientific Instruments Corp.). FTIR spectra of PUE or BCA monomer or PUE-PBCN (with or without drug) were recorded in $\mathrm{KBr}$ pellets or $\mathrm{KBr}$ cell on an FTIR spectrometer with resolution of $2 \mathrm{~cm}^{-1}$. A total of 64 scans were used and data were recorded over the range 4000$400 \mathrm{~cm}^{-1}$.

2.3.6. In Vitro Release Study. The in vitro release of PUE from the nanoparticles was studied by dialysis against phosphate buffer solutions (PBS) with 12-14 kDa molecular cutoff bag pH 7.4 [19]. PUE-PBCN suspension or PUE propylene glycol solution (containing $3 \mathrm{mg} / \mathrm{mL}$ of PUE) was placed into dialysis bags, respectively. Then the bags were suspended in flasks containing $150 \mathrm{~mL}$ of PBS as dissolution medium at $37^{\circ} \mathrm{C}$ in shaking water bath at $100 \mathrm{rpm} .1 \mathrm{~mL}$ of dissolution medium was withdrawn at regular time intervals, and the same volume was added with fresh release medium. The concentrations of PUE in dissolution medium were measured using UV spectrophotometer at $251 \mathrm{~nm}$. All experiments were performed in triplicates.

2.4. Pharmacokinetics Study. The study on the pharmacokinetics of PUE-PBCN was performed in male Wistar rats $(200 \pm 20 \mathrm{~g})$ supplied by the Medical Animal Test Center of Shandong University [20]. All animal experiments complied with the requirements of the National Act on the use of experimental animals (China). The animals were divided randomly into 2 groups $(n=5)$, housed in an environmentally controlled breeding room (temperature $25 \pm 2{ }^{\circ} \mathrm{C}$, humidity $60 \pm 5 \%, 12 \mathrm{~h}$ dark/light cycle) for 7 days and fasted overnight before experiment with free access to water. Group 1 was orally administrated PUE suspension $(30 \mathrm{mg} / \mathrm{kg}$, PUE dispersed in $0.5 \%$ sodium carboxymethylcellulose (CMC$\mathrm{Na}$ ) solution), and Group 2 was given PUE-PBCN at the same dose of $30 \mathrm{mg} / \mathrm{kg}$ through the same route. $0.3 \mathrm{~mL}$ of blood was withdrawn from the subclavian vein at $0 \mathrm{~min}$, $5 \mathrm{~min}, 10 \mathrm{~min}, 20 \mathrm{~min}, 40 \mathrm{~min}, 1 \mathrm{~h}, 2 \mathrm{~h}, 4 \mathrm{~h}, 6 \mathrm{~h}, 8 \mathrm{~h}, 12 \mathrm{~h}$, and $24 \mathrm{~h}$. The samples were placed into heparinized tubes and isolated immediately by centrifugation at $4000 \mathrm{rpm}$ for $10 \mathrm{~min}$. The plasma obtained was stored at $-20^{\circ} \mathrm{C}$ before analysis.

\subsection{Liquid Chromatography Tandem Mass Spectrometry} (LC-MS) Analysis. Extraction of PUE from plasma was conducted as follows: $25 \mu \mathrm{L}$ of internal standard solution (genistein, $1 \mu \mathrm{g} / \mathrm{mL}$ ) and $75 \mu \mathrm{L}$ of methanol were added to $25 \mu \mathrm{L}$ of plasma and the resulting mixture was vortexed for $1 \mathrm{~min}$ and then centrifuged at 11,000 rpm for $5 \mathrm{~min}$ to obtain a clean supernatant. An aliquot $(20 \mu \mathrm{L})$ of supernatant was injected into the LC-MS for analysis [21].

PUE in plasma was determined by LC-MS analysis using an Agilent 1200 system equipped with an autosampler, 

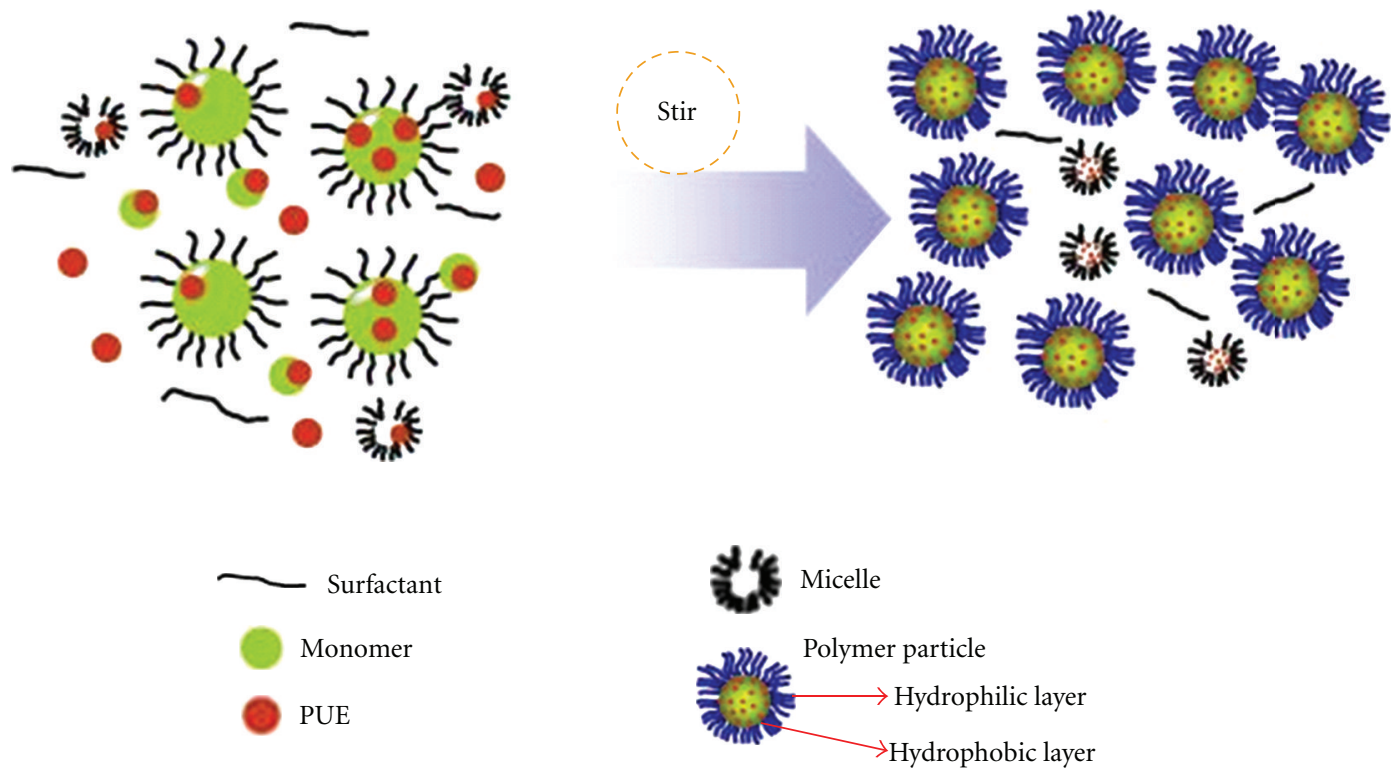

FIgURE 2: The fabrication of PUE loaded PBCN.

a vacuum degasser unit, and a binary pump. The HPLC system was coupled to an Agilent G6410B triple-quadrupole mass spectrometer (Agilent Technologies, USA), equipped with electrospray ionization (ESI) run by Agilent Mass Hunter Workstation B.01.03.

Mass spectrometric analysis was performed in multiple reaction monitoring (MRM) mode by monitoring ion transitions at $\mathrm{m} / \mathrm{z} 415.1 \rightarrow 295.1$ for PUE and $269.1 \rightarrow 133.0$ for genistein (IS), with spray gas pressure of $350 \mathrm{~Pa}$, protective air of nitrogen gas at a flow rate of $10 \mathrm{~L} / \mathrm{min}$, dwell time of $200 \mathrm{~ms}$, capillary voltage of $4000 \mathrm{~V}$, fragment electric voltage of $150 \mathrm{~V}$ for PUE and $135 \mathrm{~V}$ for genistein, and collision energy of $20 \mathrm{eV}$ for PUE and $25 \mathrm{eV}$ for genistein. Chromatographic peaks of analyte and IS were identified on the basis of retention time and MRM response.

Chromatographic separation was performed using a ProntoSIL C18 column $(150 \mathrm{~mm} \times 3 \mathrm{~mm}, 3 \mu \mathrm{m}$, Bischoff, Germany). The mobile phase consisted of a mixture of $10 \mathrm{mM}$ ammonium acetate in water containing $0.1 \%$ formic acid and methanol $(20: 80, \mathrm{v} / \mathrm{v})$. The separation was performed under isocratic conditions with a constant flow rate of $0.6 \mathrm{~mL} / \mathrm{min}$. The analytical method was validated according to "Guidance for Industry-Bioanalytical Method Validation" [22]. Pharmacokinetic parameters were evaluated using Pharmacokinetic program DAS 2.0 (supplied by Chinese Pharmacological Society).

2.6. Statistical Analysis. The statistical analysis of the data was carried out using Student's $t$-test with $P<0.05$ as the minimal level of significance.

\section{Results and Discussion}

3.1. Fabrication of PUE-PBCN. PUE-PBCN was prepared by anionic polymerization based on the reported method
[3]. Based on the reported mechanism of polymerization $[3,23]$, the PUE-PBCN may fabricate as follows (Figure 2). Firstly, the surfactant such as F68 dissolved in acidic media above the critical micelle concentration (CMC) and the monomer-swollen micelles were formed as micelles took up BCA monomer. Secondly, the anionic radicals present in the media entered the swollen structures to initiate the polymerization, resulting in the formation of the primary particles. The newly formed polymer particles continued to grow in size by absorbing monomer molecules until the monomer completely disappeared. These primary particles were stabilized by the surfactant (F68) and stabilizer (Dextran 70), which prevented the aggregation of formed particles by steric repulsion. Concurrently, PUE, a high hydrophobic drug, might be gradually dispersed in the hydrophobic part of the swollen micelles and further incorporated in polymer nanoparticles by emulsion during the polymerization. The mean encapsulation efficiency and drug loading of PUEPBCN suspension were $48.75 \%$ and $15.02 \%$, respectively.

3.2. Characterization of PUE-PBCN. As shown in Figure 3, PUE-PBCN was spherical in shape with a smooth surface under TEM, which was in consistent with the reported result of PBCN loading curcuminoids [3].

It can be seen from Figure 4 that more than $85 \%$ of PUEPBCN was between $100 \mathrm{~nm}$ to $300 \mathrm{~nm}$, indicating that the size range of $\mathrm{PBCN}$ was narrow. The mean diameter was $159.4 \mathrm{~nm}$ with polydispersity index (PDI) of 0.139 .

In the present study, the mean zeta potential of PUEPBCN was $-15.00 \mathrm{mV}$, which was similar to the report of Rivastigmine-loaded PBCN [5].

3.3. DSC and XRD Analysis. DSC, characterizing the thermal behavior of polymer and drug correlated to their melting and recrystallization, was used to investigate the physicochemical 


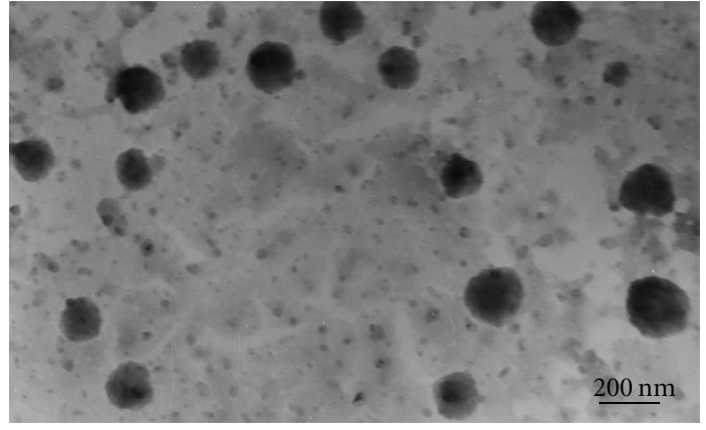

FIgURE 3: TEM photograph of PUE-PBCN.

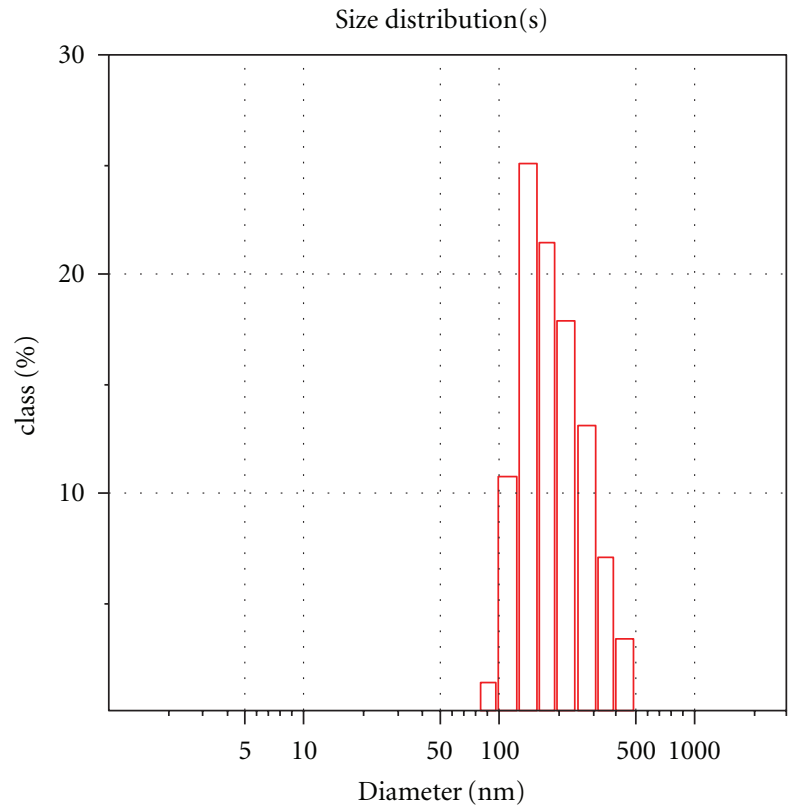

FIgUre 4: The size distribution of the PUE-PBCN.

state of PUE in the formulation [24]. The DSC thermograms of pure PUE, blank PBCN, and PUE-PBCN were shown in Figure 5. The wide endothermic peak at $112.2^{\circ} \mathrm{C}$ present in the thermogram of the crude PUE was not visible in that of PUE-PBCN, implying that PUE in PBCN was not in crystalline state but in an amorphous form. Blank PBCN showed an endothermic peak about $55^{\circ} \mathrm{C}$, while the peak disappeared in the thermogram of PUE-PBCN, which might be due to drug interfering in the heat flow [25].

XRD analysis was employed to study the potential changes of the crystalline state of PUE in PBCN. The XRD patterns for PUE-PBCN, blank PBCN, and pure PUE were shown in Figure 6. Compared with the diffractograms for blank PBCN and pure PUE, almost all diffraction peaks disappeared in that of PUE-PBCN, indicating that PUE was in an amorphous form.

3.4. FTIR Analysis. FTIR analysis was proposed to evaluate possible interactions between drug and polymer carrier. Figure 7 showed the infrared spectra of BCA monomer (a),

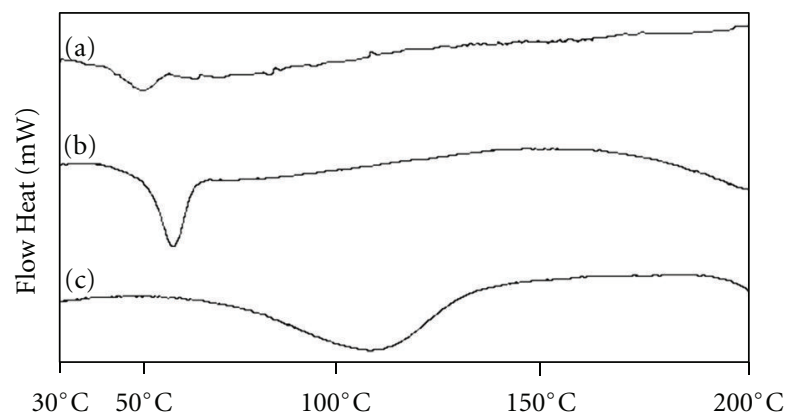

Figure 5: DSC thermograms of PUE-PBCN (a), blank PBCN (b) and pure PUE (c).

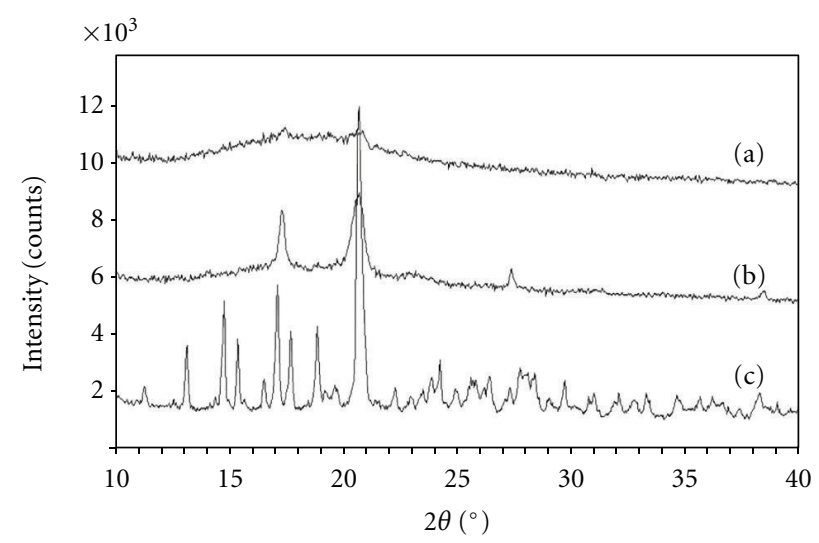

Figure 6: XRD diffractograms of PUE-PBCN (a), blank PBCN (b) and pure PUE (c).

blank PBCN (b), PUE-PBCN (c), and pure PUE (d). The peak at about $2200 \mathrm{~cm}^{-1}$ in spectra of BCA is characteristic of $\mathrm{C} \equiv \mathrm{N}$ (stretching mode of the polymer). The $\mathrm{C} \equiv \mathrm{N}$ peak was also present in the spectra of blank PBCN and PUE$\mathrm{PBCN}$, indicating that $\mathrm{C} \equiv \mathrm{N}$ did not participate in the polymerization. Broader bands at about $3400 \mathrm{~cm}^{-1}$ were observed in the spectra of blank PBCN, PUE-PBCN, and pure PUE, which were corresponding to hydroxyl radicals. Compared to the spectrum of BCA monomer, these bands in spectra of $\mathrm{PBCN}$ may be a result of polymerization, which can be inferred from the chemical structures of BCA and PBCA (Figures 1(b) and 1(c)) [25]. Moreover, the fingerprint region at $1600 \mathrm{~cm}^{-1}$ and $650 \mathrm{~cm}^{-1}$ assigned to the absorption peak of benzene did not change or shift and only got weak in the spectrum of PUE-PBCN, which could be a strong evidence showing no new chemical bond produced. All results indicated that PUE did not participate in the polymerization reaction and was only encapsulated in $\mathrm{PBCN}$ by possible intermolecular forces such as hydrogen bond. Similar results were obtained in the previous report [26].

3.5. In Vitro Release Study. The in vitro release of PUE from $\mathrm{PBCN}$ was conformed in phosphate buffer at $\mathrm{pH} 7.4$ with dynamic dialysis method. The results of drug release from PUE solution or PBCN suspension at $\mathrm{pH} 7.4$ were shown in Figure 8. The PUE release from solution was found to 


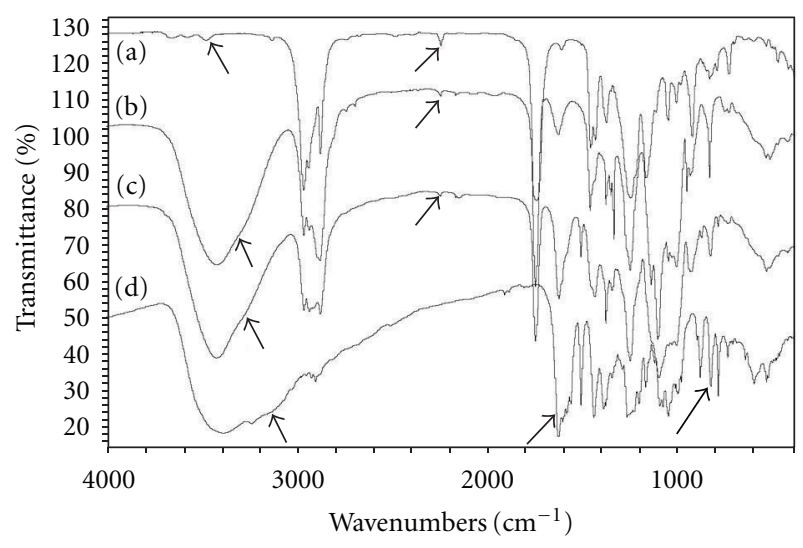

Figure 7: FTIR spectra of BCA (a), blank PBCN (b), PUE-PBCN (c) and pure PUE (d).

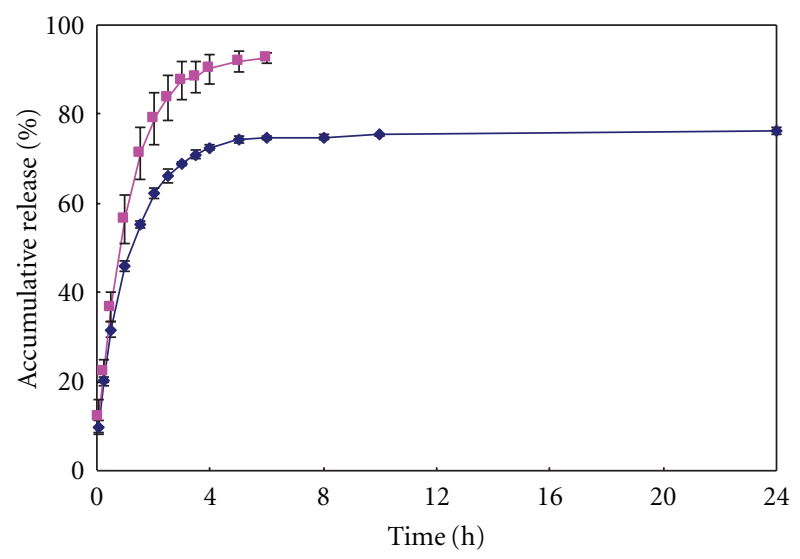

Figure 8: In vitro release profiles of PUE from $\mathrm{PBCN}$ in $\mathrm{PBS}$ at $\mathrm{pH}$ $=7.4$.

be much faster, nearly $100 \%$ in $5 \mathrm{~h}$. In contrast, the release profile of PUE from PBCN demonstrated two phases: an initial burst release followed by a sustained release, only about $75 \%$ over the period of $24 \mathrm{~h}$. This could be attributed to the fact that PUE loaded on or near the surface of PBCN was released first, and subsequently the drug embedded in the nanoparticles was released, which might release slowly by diffusion from the matrix or along with the degradation of polymer [27]. The result was in agreement with the previous reports that drug-loaded $\mathrm{PBCN}$ provided a controlled release pattern [28].

3.6. Pharmacokinetics Study. The mean concentrations of PUE in the plasma after oral administration of a single dose in rats were measured using a highly sensitive and specific LC/MS/MS method. The retention time of puerarin and genistein was $1.4 \mathrm{~min}$ and $1.8 \mathrm{~min}$, respectively. The mean recovery of PUE was more than $93.0 \%$, and the precision for interday and intraday was less than $15 \%$. Besides, PUE in analyzed samples was stable for $24 \mathrm{~h}$ at room temperature, 30 days at $-20^{\circ} \mathrm{C}$ and three freeze-thaw cycles.

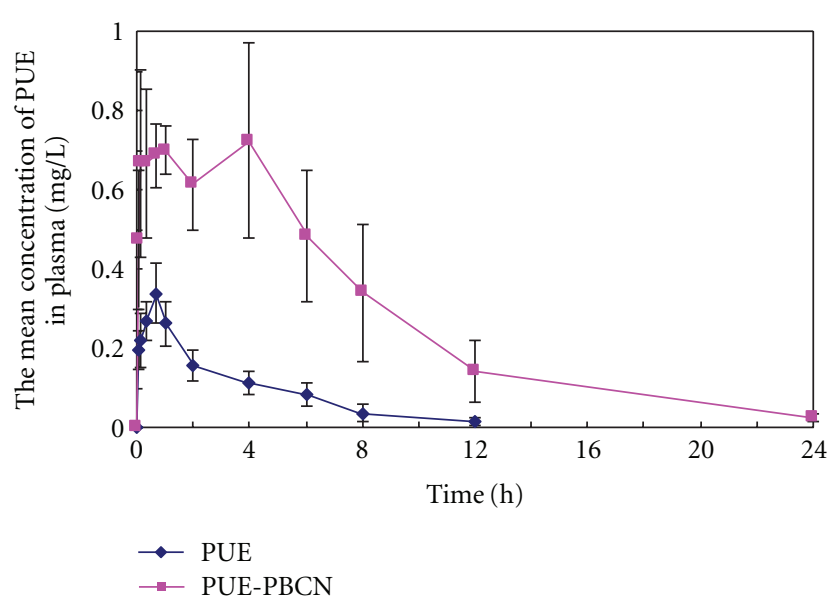

FIGURE 9: Concentration-time curves of PUE suspension and PUE$\mathrm{PBCN}$ in rats.

The calibration curve was linear over the concentration range of $2-10000 \mathrm{ng} / \mathrm{mL}$ for PUE, and the regression equation was $A=8.01 \times 10^{-5} \mathrm{C}+2.35 \times 10^{-4}$ with the mean correlation coefficient $(r)$ of 0.9996 .

The data for pharmacokinetics in rats were analyzed with DAS 2.0, and pharmacokinetic parameters were shown in Table 1. The pharmacokinetic behaviors of PUE suspension and PUE-PBCN were described using noncompartment model. As shown in Table 1, the area under the concentration-time curve (AUC) of PUE-PBCN was $6.765 \pm$ $2.374 \mathrm{mg} / \mathrm{L} * \mathrm{~h}$, which was 5.56 -fold greater than that of PUE suspension administration $(1.216 \pm 0.158 \mathrm{mg} / \mathrm{L} * \mathrm{~h}, P<$ 0.01 ). The mean residence time (MRT) of PUE-PBCN (6.299 $\pm 0.925 \mathrm{~h})$ and half-life $T_{1 / 2}(3.937 \pm 0.972)$ were 1.63 times those of CUR suspension $(3.864 \pm 0.832 \mathrm{~h}$ for MRT, $2.409 \pm 0.791 \mathrm{~h}$ for $\left.T_{1 / 2}\right)$, while the plasma clearance (4.936 $\pm 1.810 \mathrm{~L} / \mathrm{h} \cdot \mathrm{kg})$ of CUR-PBCN was much lower relative to that of the control suspension $(25.04 \pm 3.489 \mathrm{~L} / \mathrm{h} \cdot \mathrm{kg})$. These results might be related to the small size of nanoparticles and coating of F68 on the surface to keep the nanoparticles contacting closely and long with the gastrointestinal (GI) tract [29]. The mean plasma concentration-time profiles for PUE and PUE-PBCN were presented in Figure 9. At all time points, the PUE concentrations in plasma were significantly higher for rats treated with PUE-PBCN than those treated with PUE suspension. Twenty-four hours after oral administration of PUE-PBCN, the PUE concentration in plasma was still more than $0.025 \mathrm{mg} / \mathrm{L}$, while it was undetectable after $12 \mathrm{~h}$ for PUE suspension. Double peaks in the profile of PUE-PBCN may be contributed to, on the one hand, the possible enterohepatic recirculation; on the other hand, the absorption time lag after the first absorption existing due to the more time for PUE was needed to be released from PBCN and absorbed [30]. The similar phenomenon has been in agreement with the pharmacokinetic study of oral administration of PUE load SLNs [13]. These results indicated that PUE entrapped into PBCN led to the increased absorption of PUE by oral administration. 
TABLE 1: Main pharmacokinetic parameters of PUE suspension and PUE-PCBN in rats.

\begin{tabular}{|c|c|c|c|}
\hline \multirow{2}{*}{ Parameters } & \multirow{2}{*}{ Unit } & \multicolumn{2}{|c|}{ Value $($ mean $\pm \mathrm{SD})$} \\
\hline & & PUE suspension & PUE-PCBN \\
\hline $\operatorname{AUC}_{(0-\infty)}$ & $\mathrm{mg} / \mathrm{L} \cdot \mathrm{h}$ & $1.216 \pm 0.158$ & $6.765 \pm 2.374^{* *}$ \\
\hline $\operatorname{MRT}_{(0-\infty)}$ & $\mathrm{h}$ & $3.864 \pm 0.832$ & $6.299 \pm 0.925^{* *}$ \\
\hline$T_{1 / 2}$ & $\mathrm{~h}$ & $2.409 \pm 0.791$ & $3.937 \pm 0.972^{*}$ \\
\hline$V_{z}$ & $\mathrm{~L} / \mathrm{kg}$ & $85.41 \pm 21.63$ & $28.33 \pm 13.95^{* *}$ \\
\hline $\mathrm{CL}$ & $\mathrm{L} / \mathrm{h} \cdot \mathrm{kg}$ & $25.04 \pm 3.489$ & $4.936 \pm 1.810^{* *}$ \\
\hline$C_{\max }$ & $\mathrm{mg} / \mathrm{L}$ & $0.363 \pm 0.045$ & $0.897 \pm 0.135^{* *}$ \\
\hline
\end{tabular}

** Comparing to PUE suspension group, $P<0.001 ;{ }^{*}$ Comparing to PUE suspension group, $P<0.05$.

The relative bioavailability $\left(F_{\text {rel }}\right)$ was calculated as

$$
F_{\text {rel }}=\frac{\mathrm{AUC}_{\mathrm{PUE}-\mathrm{PBCN}}}{\mathrm{AUC}_{\mathrm{PUE}}} \times 100 \%
$$

The result indicated that PBCN could significantly enhance the oral bioavailability of PU (more than 5.5-fold that of PUE suspension). The enhanced bioavailability by the PBCN formulation might be a cowork of direct uptake of nanoparticles through the GI tract, increased permeability by surfactants, and decreased degradation and clearance for PUE. The particles in nanorange could be directly uptaken by the mucus and microfold cells (M cells) of the Peyer's patches and the lymphatics through the GI tract $[31,32]$. In addition, PUE could be protected by incorporation into PBCN during the absorption process [33]. The high affinity of PBCN to M cells in the GI tract and increased permeability by surfactants could result in long MRT and $T_{1 / 2}$, thus leading to the enhanced bioavailability $[34,35]$. The results support that $\mathrm{PBCN}$ is a promising delivery system for improving oral bioavailability of PUE.

\section{Conclusions}

In the present study, PUE-PBCN was successfully prepared to improve the bioavailability. The drug-loaded PBCN was sphere-like shape with a mean diameter of $159.4 \mathrm{~nm}$ and the zeta potential was $-15.00 \mathrm{mV}$. It could be concluded from DSC, FTIR, and X-ray analysis that PUE loaded in nanoparticles was in an amorphous form. The pharmacokinetic data showed that the PBCN could improve the oral bioavailability of PUE by 5.5 -fold. It can be concluded that PBCN can improve the oral absorption of PUE.

\section{Acknowledgments}

This work was supported by the Natural Science Foundation of Shandong Province, China (Grant no. Q2007C13), the Shandong Administration of Traditional Chinese Medicine (Grant no. 2009-152), and the Science and Technology Research Project From Population and Family Planning Commission of Shandong Province, China. L. Zhao, A. Liu and M. Sun contributed equally to the work.

\section{References}

[1] A. Dolenc, J. Kristl, S. Baumgartner, and O. Planinšek, "Advantages of celecoxib nanosuspension formulation and transformation into tablets," International Journal of Pharmaceutics, vol. 376, no. 1-2, pp. 204-212, 2009.

[2] M. Kakran, N. G. Sahoo, L. Li et al., "Fabrication of drug nanoparticles by evaporative precipitation of nanosuspension," International Journal of Pharmaceutics, vol. 383, no. 1-2, pp. 285-292, 2010.

[3] R. Mulik, K. Mahadik, and A. Paradkar, "Development of curcuminoids loaded poly(butyl) cyanoacrylate nanoparticles: physicochemical characterization and stability study," European Journal of Pharmaceutical Sciences, vol. 37, no. 3-4, pp. 395-404, 2009.

[4] C. Vauthier and K. Bouchemal, "Methods for the preparation and manufacture of polymeric nanoparticles," Pharmaceutical Research, vol. 26, no. 5, pp. 1025-1058, 2009.

[5] S. A. Joshi, S. S. Chavhan, and K. K. Sawant, "Rivastigmineloaded PLGA and PBCA nanoparticles: preparation, optimization, characterization, in vitro and pharmacodynamic studies," European Journal of Pharmaceutics and Biopharmaceutics, vol. 76, no. 2, pp. 189-199, 2010.

[6] W. He, X. Jiang, and Z. R. Zhang, "Preparation and evaluation of poly-butylcyanoacrylate nanoparticles for oral delivery of thymopentin," Journal of Pharmaceutical Sciences, vol. 97, no. 6, pp. 2250-2259, 2008.

[7] G. Fontana, G. Pitarresi, V. Tomarchio, B. Carlisi, and P. L. San Biagio, "Preparation, characterization and in vitro antimicrobial activity of ampicillin-loaded polyethylcyanoacrylate nanoparticles," Biomaterials, vol. 19, no. 11-12, pp. 10091017, 1998.

[8] C. Damgé, C. P. Reis, and P. Maincent, "Nanoparticle strategies for the oral delivery of insulin," Expert Opinion on Drug Delivery, vol. 5, no. 1, pp. 45-68, 2008.

[9] M. P. Desai, V. Labhasetwar, G. L. Amidon, and R. J. Levy, "Gastrointestinal uptake of biodegradable microparticles: effect of particle size," Pharmaceutical Research, vol. 13, no. 12, pp. 1838-1845, 1996.

[10] A. Yu, H. Wang, J. Wang et al., "Formulation optimization and bioavailability after oral and nasal administration in rabbits of puerarin-loaded microemulsion," Journal of Pharmaceutical Sciences, vol. 100, no. 3, pp. 933-941, 2011.

[11] D. Q. Quan, G. X. Xu, and X. G. Wu, "Studies on preparation and absolute bioavailability of a self-emulsifying system containing puerarin," Chemical and Pharmaceutical Bulletin, vol. 55, no. 5, pp. 800-803, 2007.

[12] H. Wu, A. Zhou, C. Lu, and L. Wang, "Examination of lymphatic transport of puerarin in unconscious lymph ductcannulated rats after administration in microemulsion drug 
delivery systems," European Journal of Pharmaceutical Sciences, vol. 42, no. 4, pp. 348-353, 2011.

[13] C.-F. Luo, M. Yuan, M.-S. Chen et al., "Pharmacokinetics, tissue distribution and relative bioavailability of puerarin solid lipid nanoparticles following oral administration," International Journal of Pharmaceutics, vol. 410, no. 1-2, pp. 138-144, 2011.

[14] N. Behan, C. Birkinshaw, and N. Clarke, "Poly n-butyl cyanoacrylate nanoparticles: a mechanistic study of polymerisation and particle formation," Biomaterials, vol. 22, no. 11, pp. 1335-1344, 2001.

[15] C. Vauthier, C. Dubernet, E. Fattal, H. Pinto-Alphandary, and P. Couvreur, "Poly(alkylcyanoacrylates) as biodegradable materials for biomedical applications," Advanced Drug Delivery Reviews, vol. 55, no. 4, pp. 519-548, 2003.

[16] L. Cai, G. Niu, Z. Hu, W. Jin, J. Wang, and L. Sun, "Polybutylcyanoacrylate magnetic nanoparticles as carriers of adriamycin," Journal of Drug Targeting, vol. 17, no. 3, pp. 200206, 2009.

[17] L. J. Jia, D. R. Zhang, Z. Y. Li et al., "Preparation and characterization of silybin-loaded nanostructured lipid carriers," Drug Delivery, vol. 17, no. 1, pp. 11-18, 2009.

[18] Q. Lv, A. Yu, Y. Xi et al., "Development and evaluation of penciclovir-loaded solid lipid nanoparticles for topical delivery," International Journal of Pharmaceutics, vol. 372, no. 1-2, pp. 191-198, 2009.

[19] A. Bayat, B. Larijani, S. Ahmadian, H. E. Junginger, and M. Rafiee-Tehrani, "Preparation and characterization of insulin nanoparticles using chitosan and its quaternized derivatives," Nanomedicine, vol. 4, no. 2, pp. 115-120, 2008.

[20] C. F. Luo, M. Yuan, M. S. Chen, S. M. Liu, and H. Ji, "Metabolites of puerarin identified by liquid chromatography tandem mass spectrometry: similar metabolic profiles in liver and intestine of rats," Journal of Chromatography B, vol. 878, no. 3-4, pp. 363-370, 2010.

[21] Y. Z. Gu, W. Zhou, and G. X. Zhai, "Preparation of puerarin liposome and its oral absorption in rat," Journal of Chinese Medicinal Materials, vol. 30, no. 8, pp. 970-973, 2007.

[22] US Food and Drug Administration, "Guidance for Industry: Bioanalytical Method Validation,” 2001, http://www.fda.gov/ downloads/Drugs/GuidanceComplianceRegulatoryInformation/Guidances/UCM070107.pdf.

[23] R. S. R. Murthy and L. H. Reddy, "Poly (alkyl cyanocrylate) nanoparticles for delivery of anti-cancer drugs," in Nanotechnology, M. M. Amiji, Ed., pp. 251-288, CRC Press, Boca Raton, Fla, USA, 2007.

[24] B. Sarmento, D. Ferreira, F. Veiga, and A. Ribeiro, "Characterization of insulin-loaded alginate nanoparticles produced by ionotropic pre-gelation through DSC and FTIR studies," Carbohydrate Polymers, vol. 66, no. 1, pp. 1-7, 2006.

[25] M. Sun, Y. Gao, C. Guo et al., "Enhancement of transport of curcumin to brain in mice by poly(n-butylcyanoacrylate) nanoparticle," Journal of Nanoparticle Research, vol. 12, no. 8, pp. 3111-3122, 2010.

[26] M. Simeonova, G. Ivanova, V. Enchev et al., "Physicochemical characterization and in vitro behavior of daunorubicin-loaded poly(butylcyanoacrylate) nanoparticles," Acta Biomaterialia, vol. 5, no. 6, pp. 2109-2121, 2009.

[27] F. Ren, R. Chen, Y. Wang, Y. Sun, Y. Jiang, and G. Li, "Paclitaxel-loaded poly(n-butylcyanoacrylate) nanoparticle delivery system to overcome multidrug resistance in ovarian cancer," Pharmaceutical Research, vol. 28, no. 4, pp. 897-906, 2011.
[28] L. H. Reddy and R. R. Murthy, "Influence of polymerization technique and experimental variables on the particle properties and release kinetics of methotrexate from poly(butylcyanoacrylate) nanoparticles," Acta Pharmaceutica, vol. 54, no. 2, pp. 103-118, 2004.

[29] J. Liu, J. Zhu, Z. Du, and B. Qin, "Preparation and pharmacokinetic evaluation of Tashinone IIA solid lipid nanoparticles," Drug Development and Industrial Pharmacy, vol. 31, no. 6, pp. 551-556, 2005.

[30] Y. Li, W. S. Pan, S. L. Chen, H. X. Xu, D. J. Yang, and A. S. C. Chan, "Pharmacokinetic, tissue distribution, and excretion of puerarin and puerarin-phospholipid complex in rats," Drug Development and Industrial Pharmacy, vol. 32, no. 4, pp. 413422, 2006.

[31] A. E. Gulyaev, S. E. Gelperina, I. N. Skidan, A. S. Antropov, G. Y. Kivman, and J. Kreuter, "Significant transport of doxorubicin into the brain with polysorbate 80- coated nanoparticles," Pharmaceutical Research, vol. 16, no. 10, pp. 1564-1569, 1999.

[32] N. Hussain, V. Jaitley, and A. T. Florence, "Recent advances in the understanding of uptake of microparticulates across the gastrointestinal lymphatics," Advanced Drug Delivery Reviews, vol. 50, no. 1-2, pp. 107-142, 2001.

[33] A. Dembri, M. J. Montisci, J. C. Gantier, H. Chacun, and G. Ponchel, "Targeting of 3'-azido 3'-deoxythymidine (AZT)-loaded poly(isohexylcyanoacrylate) nanospheres to the gastrointestinal mucosa and associated lymphoid tissues," Pharmaceutical Research, vol. 18, no. 4, pp. 467-473, 2001.

[34] D. Duchêne and G. Ponchel, "Bioadhesion of solid oral dosage forms, why and how?" European Journal of Pharmaceutics and Biopharmaceutics, vol. 44, no. 1, pp. 15-23, 1997.

[35] N. Venkatesan, K. Uchino, K. Amagase, Y. Ito, N. Shibata, and K. Takada, "Gastro-intestinal patch system for the delivery of erythropoietin," Journal of Controlled Release, vol. 111, no. 1-2, pp. 19-26, 2006. 

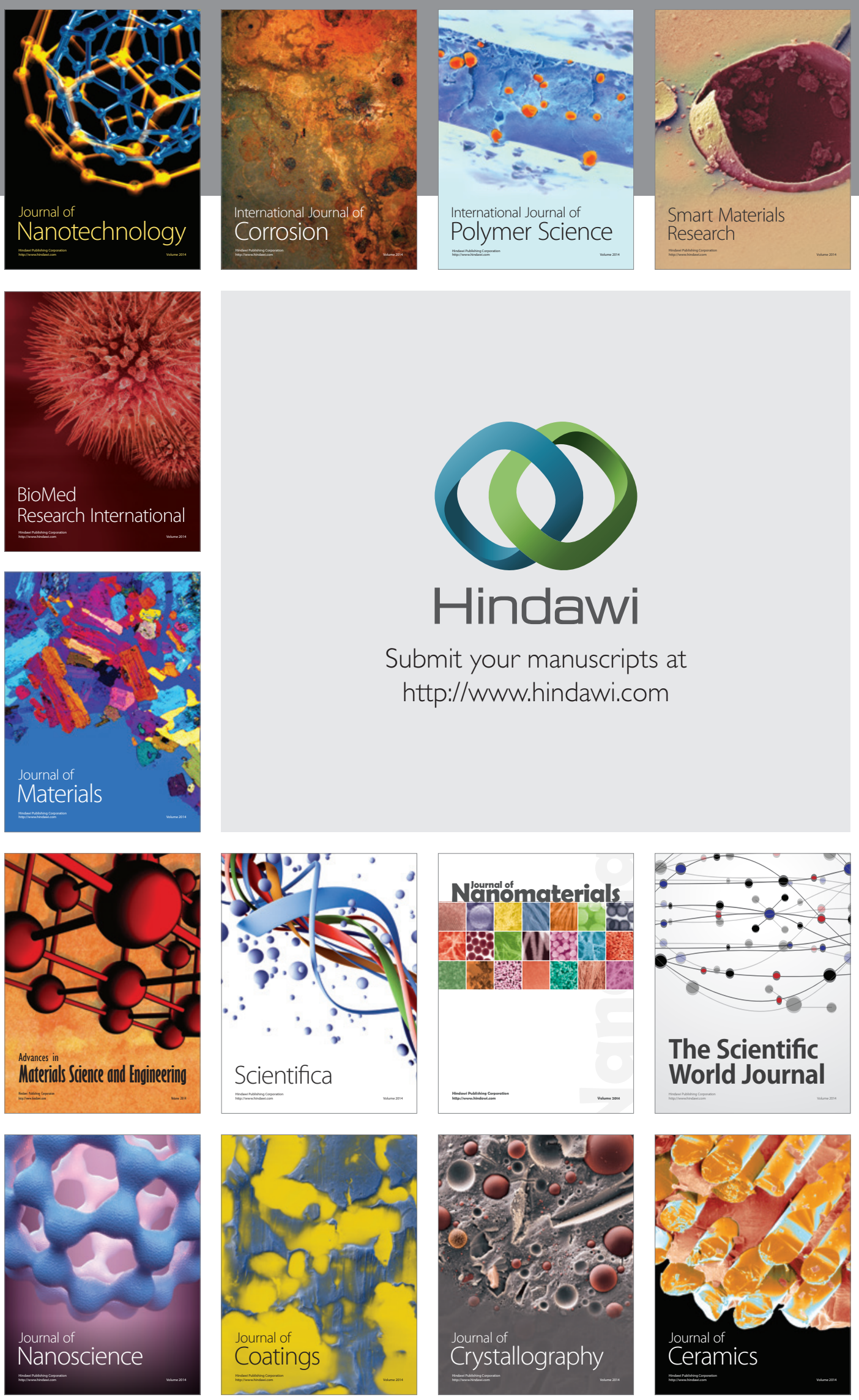

The Scientific World Journal

Submit your manuscripts at

http://www.hindawi.com

\section{World Journal}

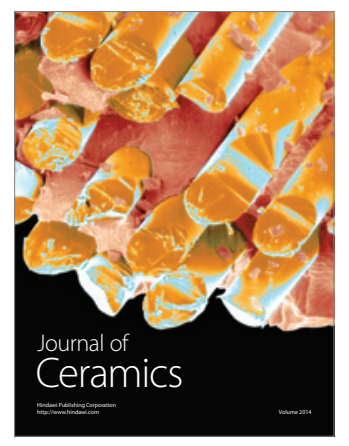

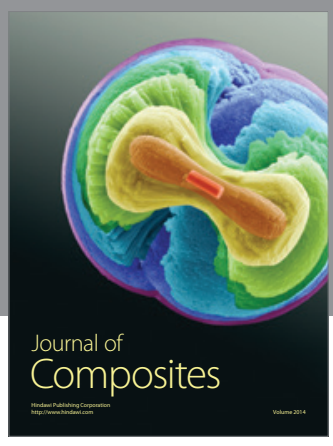
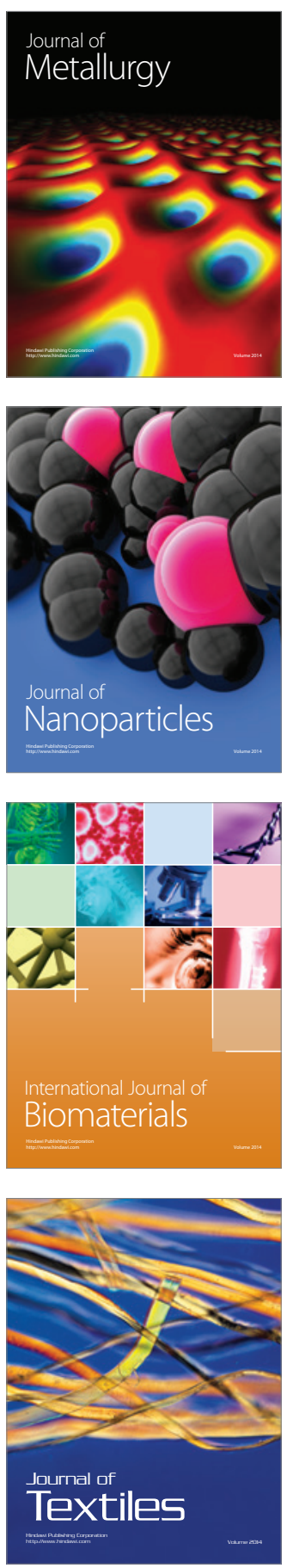